\title{
Robust platform for water harvesting and directional transport
}

\author{
Hu Luo a, b, Yao Lu ${ }^{c}$, Shaohui Yin ${ }^{\text {d }}$, Shuai Huang a, b* ,Jinlong Song ${ }^{\text {e }}$, Faze Chen ${ }^{\text {e }}$, Fengjun Chen a, b, Claire J. Carmalt ${ }^{\mathrm{f}}$, Ivan P. \\ Parkin ${ }^{\mathrm{f}}$ \\ a State Key Laboratory of Advanced Design and Manufacturing for Vehicle Body, Hunan University, Changsha, Hunan, 410082, China \\ ${ }^{b}$ College of Mechanical and Vehicle Engineering, Hunan University, Changsha, Hunan, 410082, China \\ ${ }^{c}$ Department of Mechanical Engineering, University College London, Torrington Place, London WC1E 7JE, UK \\ ${ }^{d}$ Hunan Provincial Key Laboratory of Intelligent Laser Manufacturing, Hunan University, Changsha, 410082, China \\ ${ }^{e}$ Key Laboratory for Precision \& Non-traditional machining of Ministry of Education, Dalian University of Technology, Dalian, \\ 116024, China \\ ${ }^{f}$. Materials Chemistry Research Centre, Department of Chemistry, University College London, 20 Gordon Street, London, WC1H 0AJ, \\ $U K$.
}

\begin{abstract}
Water harvesting is used for transforming moisture into available water resources in regions that suffer water scarcity. However, it remains a considerable challenge to design a system that functions to both collect water from the air and transport it to a certain region over a long distance. In this work, a new water harvesting platform for dropwise condensation and dropwise transportation is developed to realize both water collection and spontaneously directional transport over long-distance at low temperatures. The water harvesting platform was developed based on a biomimetic slippery liquid-infused porous surface (SLIPS) with micron-size steps and nanosized holes through electrochemical etching, electrochemical anodizing, low surface energy modification and lubricant infusion. The anti-wetting, chemical resistance, condensation and anti-icing properties of the water harvesting platform at different $\mathrm{pHs}$ and temperatures were tested to show the stability of the system. The water harvesting platform exhibited excellent dropwise condensation capacity, and can directly and continuously capture moisture from the air in a low-temperature environment. Spontaneously directional transport of droplets was achieved on a choreographed wedged-platform driven by Laplace pressure, and the transport distance was unlimited due to the energy conversion from surface energy to kinetic energy induced by droplets coalescing in a repeated motion of droplet merging and chasing. This water harvesting platform shows great potential in applications for advanced transportation devices, multifunctional sensors, actuators, and is a promising potential solution to water scarcity.
\end{abstract}

Key Words: Water Harvesting, Directional Transport, Condensation, Slippery Surface

\section{Introduction}

With the growth of the world economy and the increase of population, human demand for water continues to increase. This coupled with the existence of unreasonable exploitation and utilization of water resources, means that many countries and regions are facing water scarcity. Fog harvesting could transform the hidden water resources from the air into an available water resource at low cost. Inspired by natural plants and creatures with water collection ability from air, such as cactus spines ${ }^{1-3}$, desert beetles ${ }^{4-7}$, spider silks ${ }^{8,9}$, mimic multi-function-systems with fog harvesting and vapor condensation abilities have attracted increasing attention in both fundamental research and in many technological innovations. After several underlying theories have been developed, the water collection ability from the air in nature is mainly attributed to the unique wettability or special structural topography, which enhances the condensation efficiency of water. Currently, biomimetic surfaces with water collection ability have 
promising applications in antifreeze, thermal management, self-cleaning, enhanced condensation heat transfer and energy harvesting. ${ }^{10-13}$ Song et al. ${ }^{14-16}$ developed a superhydrophobic-superhydrophilic patterned surface for water harvesting, where high condensation and water collection efficiency were realized by the unique wettability of a substrate. In the case of a special structural topography, Wang et al. ${ }^{9}$ fabricated a spindle-knot microfiber and designed a spider-web-like network to obtain large-scale and highly efficient water collection from a foggy atmosphere. Generally, there are two forms of water condensation of moisture from the air: filmwise condensation and dropwise condensation. ${ }^{17-19}$ Filmwise condensation refers to the condensate liquid forming a continuous film, while dropwise condensation refers to the harvesting of condensate liquids in the form of droplets. In consideration of heat-transfer efficiency during phase-change, dropwise condensation is over ten times more than that of filmwise condensation, and the heat-transfer performance of dropwise condensation can be further improved by fabricating micro/nano-structures on the substrate. ${ }^{20-22}$ Therefore, compared with the filmwise condensation, the dropwise condensation is more applicable for high-efficiency water harvesting, and it can also reduce the residue liquid on the substrate. However, condensation alone is not able to achieve the full functions of water harvesting because directional transport of water is also required. For example, cactus spines not only collect moisture from the air, but also transport collected water to the body of cactus. Therefore, directional transport of water should also be taken into consideration.

Spontaneous directional transport of water has become increasingly popular in the fields of microfluidic analysis, advanced devices design, and energy-saving. This phenomenon was discovered on the peristome surface of the Nepenthes alata plant. ${ }^{23-27}$ Artificial system for directional water transport has also been reported including wettability patterning device, superhydrophobic pump, and thermal/geometrical/chemical gradient driven approaches. ${ }^{28-32}$ Numerous mechanisms could be used to design an artificial fluid-transport system to realize spontaneous directional transport of liquids, but the types were almost always filmwise transport. However, filmwise transportation becomes ineffective because it is inevitable that the water evaporates rapidly at high temperature or freezes quickly at low temperature when the liquid spreads into a thin film. Wang et $\mathrm{l}^{33}$ fabricated a topographically patterned surface to obtain directional transport of a water droplet as a bulk movement at high temperature. Cheng et al ${ }^{34}$ designed a shape gradient surface for dropwise transportation of water at room temperature. Compared with filmwise transportation, dropwise transportation effectively avoids rapid evaporation or freezing, and reduces the residual liquid on the transport device. Hence, dropwise transportation is a better method for spontaneous transportation of the condensed water, especially at low temperature. Additionally, although some pioneering work has reported spontaneous transport of water droplet, the transport distance is only from a few millimeters to centimeter scales $28,31,35$, which invariably limits their practical application. It remains a considerable challenge to design an integrated system to collect water from air, and spontaneously transport the collected water to the desired region by dropwise transportation over a long-distance.

Here, we report a water harvesting platform for water collection from air and the spontaneous directional transport of the collected droplet based on a biomimetic slippery liquid infused porous surface (SLIPS) at low temperature. The water harvesting platform was fabricated by electrochemical etching, electrochemical anodizing, low surface energy modification followed by lubricant infusion. The key factors of anti-wetting, condensation and anti-icing properties at different $\mathrm{pH}$ values and freezing environments were investigated. The water harvesting platform could directly and continuously capture the moisture from the air to form water droplets, which could be directionally transported over a comparatively long-distance being driven by droplet merging and chasing. Finally, a multifunctional water harvesting platform which contained a collection region, transportation region and reservoir region was designed to realize dropwise condensation and dropwise transportation, achieving both dropwise water harvesting and spontaneous directional transport.

\section{Experimental Section}


Materials. Aluminum plates (purity $\geq 99 \%$; thickness, $2 \mathrm{~mm}$ ) were purchased from the Changsha Mental Material Manufacturer (China). $\mathrm{NaCl}$ and $\mathrm{H}_{2} \mathrm{C}_{2} \mathrm{O}_{4}$ (analytical purity) were purchased from Tianjin Kermel Chemical Reagent Co. (China). Fluoroalkylsilane [FAS, $\left.\mathrm{C}_{8} \mathrm{~F}_{13} \mathrm{H}_{4} \mathrm{Si}\left(\mathrm{OCH}_{2} \mathrm{CH}_{3}\right)_{3}\right]$ (analytical purity) was purchased from Jianyi Chemical Co. (China). Silicone oil ( $20 \mathrm{mPa} . \mathrm{s}$ at $\left.25{ }^{\circ} \mathrm{C}\right)$ was purchased from Aladdin Co. (China).

Fabrication of the SLIPS. The pure aluminum plate was electrochemically etched (anode, aluminum plate; cathode, copper plate) for $7 \mathrm{~min}$ in $0.1 \mathrm{~mol} / \mathrm{L} \mathrm{NaCl}$ neutral electrolyte at an electric current density of $0.5 \mathrm{~A} / \mathrm{cm}^{2}$, and the etched surface then became superhydrophilic. The superhydrophilic surface was treated with anodic oxidation in $0.3 \mathrm{~mol} / \mathrm{L}$ oxalic acid $\left(\mathrm{H}_{2} \mathrm{C}_{2} \mathrm{O}_{4}\right)$ aqueous solution under a constant voltage of $60 \mathrm{~V}$, and dense nano-sized holes were then formed on the etched Al plate surface. Subsequently, the anodized surface was immersed in 1\% FAS solution for $40 \mathrm{~min}$, then dried at $100{ }^{\circ} \mathrm{C}$ for $20 \mathrm{~min}$. Finally, silicone oil was added to the superhydrophobic surface, and the lubricated surface was inclined at $45^{\circ}$ for $2 \mathrm{~h}$ to remove excess lubricants, to form the SLIPS surfaces.

Characterization. An optical contact angle meter (HDCA001, China), scanning electron microscope (SEM, SUPRA 55 SAPPHIRE, Germany), and energy-dispersive X-ray spectroscopy (EDS, SUPRA 55 SAPPHIRE, Germany) were used to measure the water contact angle (CA), microscopic structural topography, and chemical composition of the prepared samples, respectively. Anti-wetting tests. The anti-wetting property of the water harvesting platform was observed by studying the droplet impact phenomenon on the surface using a high-speed camera (SONY RX100V, Japan) at an environment temperature of $25{ }^{\circ} \mathrm{C}$. The droplet volume was $\sim 5 \mu \mathrm{L}$. The temperatures of the tested samples were $0{ }^{\circ} \mathrm{C}, 27{ }^{\circ} \mathrm{C}$ and $100{ }^{\circ} \mathrm{C}$, respectively. The distance between droplets and samples was $50 \mathrm{~mm}$.

Condensation and anti-icing test. The condensation experiment was performed at an environment temperature of $25{ }^{\circ} \mathrm{C}$, and a semiconductor refrigeration device (temperature, $-2 \sim 1{ }^{\circ} \mathrm{C}$ ) was employed to cool the test samples. To test the anti-icing property, the water harvesting platform and ordinary $\mathrm{Al}$ plate were placed in a low temperature test chamber with a temperature of $-15{ }^{\circ} \mathrm{C}$ and $-30{ }^{\circ} \mathrm{C}$, and the ice of a volume of $2 \times 2 \times 2 \mathrm{~cm}^{3}$ was formed on the sample with the assistance of the mold, then the ice was removed using a force gauge with data acquisition frequency of $0.1 \mathrm{~s}$ and resolution of $0.1 \mathrm{~N}$ (HP-100, Handpi Co.) at an environment temperature of $25^{\circ} \mathrm{C}$, meanwhile the deicing force was automatically recorded during the ice removal process.

Directional Transport of Droplets on the water harvesting platform. In the directional transport experiment, the water harvesting platform was cut into a wedge structure by a linear cutting machine. The experiment was performed at an environment temperature of $25{ }^{\circ} \mathrm{C}$, and the test samples were cooled to $-2 \sim 1{ }^{\circ} \mathrm{C}$ by the semiconductor refrigeration device powered by electricity whose power rating is $120 \mathrm{~W}$ and voltage rating is $12 \mathrm{~V}$. The fog atmosphere was provided by a household humidifier with a humidifying capacity of $\sim 300 \mathrm{~mL} / \mathrm{h}$.

\section{Results and Discussion}

\subsection{Characterization of the slippery liquid infused porous surfaces}

As shown in Fig. 1 (a), the fabrication process of the SLIPS mainly consists of electrochemical etching, electrochemical anodizing, FAS modification and lubricant infusion. 

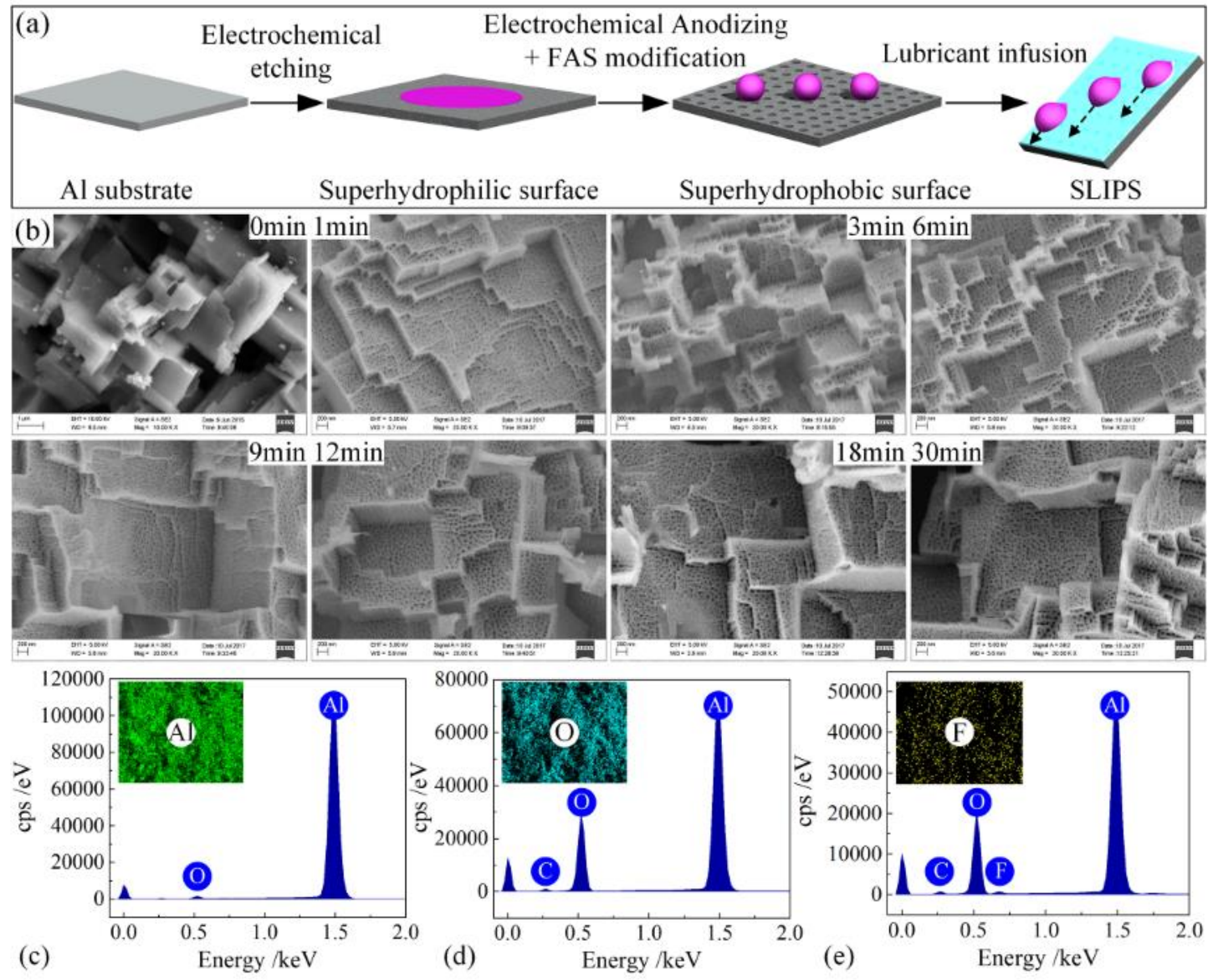

Fig. 1 (a) Fabrication of SLIPS by electrochemical etching, electrochemical anodizing, FAS modification and lubricant infusion. (b) SEM images of the electrochemical anodized surface at different anodized times. (c-e) EDS spectra of the etched surface, the anodized surface and the superhydrophobic surface (the insert graph is element mapping).

After electrochemical etching, the Al surface was covered by the micron-sized stepped structure, as shown in Fig. 1 (b). After anodic oxidation for $1 \mathrm{~min}$, the stepped structure was retained, and a large amount of regular nano-sized holes appeared. At longer anodizing times, these holes became more apparent and uniform, with their diameters gradually increasing. However, the stepped structures were not damaged. The optimal time duration for anodic oxidation was determined by the further study of the sliding angles (SA).

The element composition and distribution of the prepared surface are shown in Fig. 1 (c-e). The electrochemically etched surface mainly consists of aluminum, with a lower content of oxygen [Fig. 1 (c)]. After the electrochemical anodizing, the anodized surface consists of not only aluminum but also a large amount of oxygen [Fig. 1 (d)]. According to element mapping, oxygen was uniformly distributed on the oxidized surface. After FAS modification, fluorine was observed on the surface [Fig. 1 (e)], which means the F-containing low surface energy functional groups of FAS were successively assembled onto the surface, and the prepared surface showed superhydrophobicity. The micron steps and nano-sized holes on the superhydrophobic surface were infused with silicone oil, to form a lubrication film that functioned as a bioinspired slippery liquid infused porous surface.

\subsection{Anti-wetting Property and Chemical Stability}

Fig. 2 (a) shows the water contact angle (WCA) $\theta$ and SA of the prepared SLIPS at different anodized times during the preparation process. When the anodized time was 1-30 min, the WCAs generally varied within 100$110^{\circ}$ and SA was lower than $5^{\circ}$. When the anodized time was 6-9 min, SA was minimal, around $2^{\circ}$. To understand the chemical resistance properties of the surface, $\mathrm{H}_{3} \mathrm{PO}_{4}(\mathrm{pH}=1)$, water $(\mathrm{pH}=7)$, and $\mathrm{NaOH}(\mathrm{pH}=13)$ were 
positioned onto the SLIPS $\left(10^{\circ}\right.$ tilted), respectively, as shown in Fig. 2 (b). All the droplets slid off, indicating that the prepared SLIPS has good acid and alkali resistance.

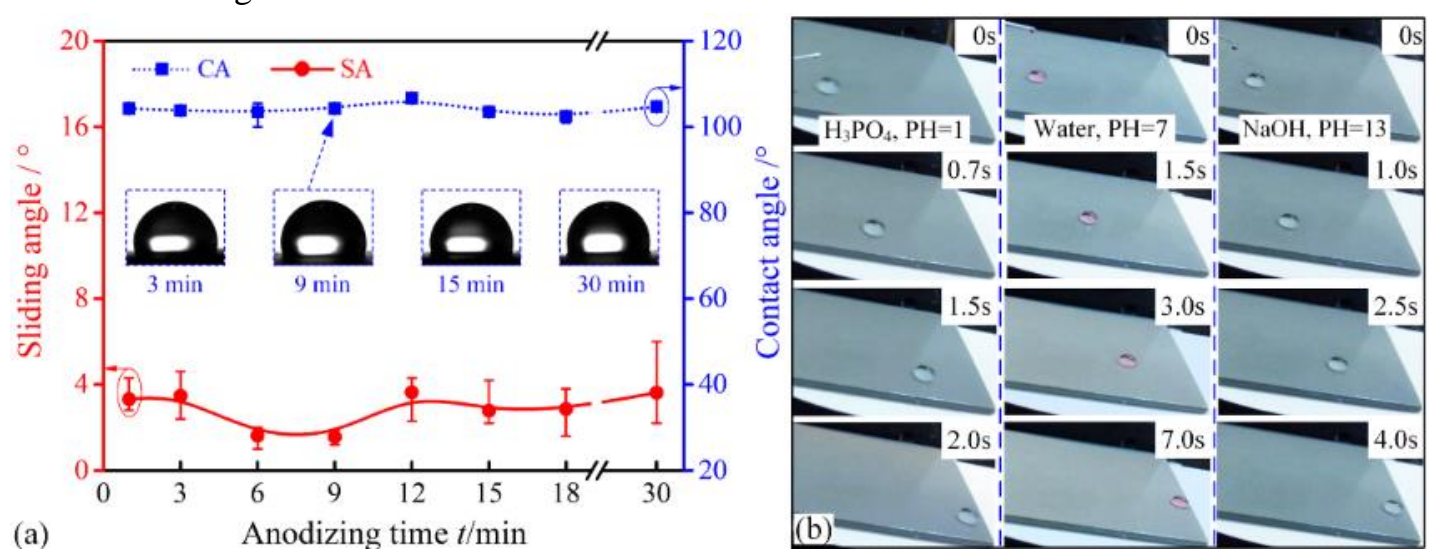

Fig. 2 (a) Variation in the contact angle and sliding angle with anodizing time. (b) Time-lapse images showing the sliding process of water droplets with different $\mathrm{pHs}$.

The anti-wetting property, especially the performance against dynamic droplets in rainy or dewy weather, is significant for the practical applications of water harvesting and transport. To understand the dynamics of water droplet on the SLIPS at different temperatures, we performed droplet impacting experiments on SLIPS at $0{ }^{\circ} \mathrm{C}$, $27{ }^{\circ} \mathrm{C}$ and $100{ }^{\circ} \mathrm{C}$ respectively, as shown in Fig. S1 (a)-(c). The results show that the phenomenon of bouncing was observed on the SLIPS, and the droplet was split during the bouncing process. With the increase of surface temperature, the droplet had a transition from a partial re-bounce to a complete re-bounce. Furthermore, when water droplet impacted on a $15^{\circ}$ tilted SLIPS, the droplet bounced and slid along the inclined surface [Fig. S1 (d)]. Continuous water jet was also introduced to further test the anti-wetting property of the SLIPS [Fig. S1 (e) and Movie S1]. The experimental results show that after the water jet impacts on the SLIPS, it did not bounce back from SLIPS, but immediately slid on SLIPS.

According to the above analysis, the prepared SLIPS has excellent anti-wetting properties and chemical resistance to repel both acidic and alkaline droplets. The surfaces are able to repel water at different temperatures and retain water repellent properties even under a strong water jet impact.

\subsection{Condensation}

To collect water from air at low temperature, the moisture condensation and anti-icing properties should be taken into consideration. For the ordinary aluminum plate with a temperature of $-2 \sim 1{ }^{\circ} \mathrm{C}$, the moisture in the air quickly condensed and nucleated, and then formed a visible water film that covered the whole surface after 2 min [Fig. 3 (a)]. At $21 \mathrm{~min}$, the water film on the surface was frozen into a frost/ice state. At $40 \mathrm{~min}$, the frost/ice showed clear boundaries. At 2 hours, the surface was fully covered by the ice layer. Therefore, the moisture in the air was in a form of filmwise condensation when contacted with an ordinary Al plate and then became a water film, and finally aggregated into frost/ice at low temperature [Fig. 3 (b)]. Meanwhile, the SLIPS adopted a different strategy to achieve condensation. For the SLIPS on a cooling device at a temperature of $-2 \sim 1{ }^{\circ} \mathrm{C}$, the moisture in the air quickly condensed, nucleated, and formed tiny water droplets, rather than a water film [Fig. 3 (c)]. At 2 min, the droplet with a tiny volume stacked on SLIPS, and could hardly slide off. At $19 \mathrm{~min}$, with an increase of droplet volume, the droplet started to slide along the inclined SLIPS driven by gravitation. During the sliding process, the collected droplets coalesced with each other [Fig. 3 (d)]. Arising from the increasing volume, the droplets finally slid off the SLIPS. In 2 hours, frost/ice was not observed on the SLIPS. Therefore, the SLIPS has excellent dropwise condensation and anti-icing property, which can be used to collect moisture in the air directly and continuously, then allow them to coalesce into droplets and slide off.

We then designed a water harvesting platform based on SLIPS to investigate the water collection efficiency [Fig. 
3 (e)]. Results show that the water collection efficiency increased with humidity. When the relative humidity was $90 \%$, the water collection efficiency exceeded $1.2 \mathrm{mg} /\left(\mathrm{cm}^{-2} \cdot \mathrm{min}\right)$.
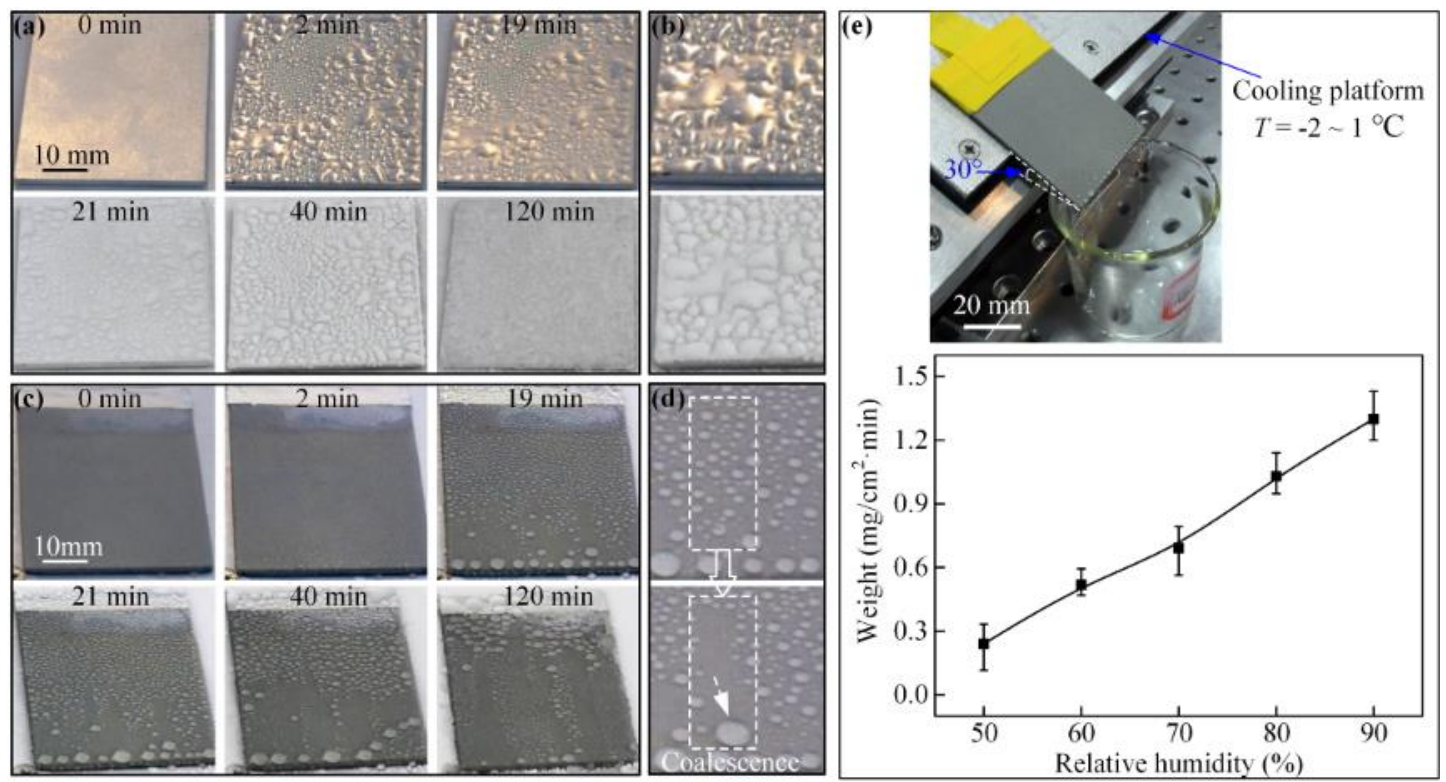

Fig. 3 Condensation property of (a, b) ordinary surface and (c, d) SLIPS on a cooling device at a temperature of $2 \sim 1{ }^{\circ} \mathrm{C}$. Where (b) and (d) are the enlarged pictures of condensation process in (a) and (c), respectively. (e) Water weight during water collection on water harvesting platform at different relative humidity.

SLIPS showed perfect dropwise condensation and anti-icing properties in a freezing environment (Supporting Information: S2). SLIPS could directly and continuously capture the moisture from the air and collect water droplets, wherein the water collection efficiency increased with humidity. As the collected water droplet volume increased, the droplet would slip along the surface in the direction of the inclined surface under the action of gravity and would encourage further coalescence with each other. Under the temperature of $-5{ }^{\circ} \mathrm{C}$, the droplets impacted on SLIPS and quickly froze into ice particles. These ice particles slid down along the surface, rather than froze into ice blocks on the surface [Fig. S2 (a)]. Even though staying at $-15{ }^{\circ} \mathrm{C}$ for 4 hours, the ice blocks could still be easily removed by a small deicing force without leaving any ice residue [Fig. S2 (c)-(g)].

\subsection{Directional Transport of Droplets on Water Harvesting Platform}

The above results show that the SLIPS were able to collect water from the air at low temperature. To achieve a complete water harvesting system, collected water is expected to be delivered to certain areas, and this requires transport of water. To transport the collected water droplet directionally, the water harvesting platform was designed into a wedge structure based on SLIPS [Fig. 4 (a)]. The water droplet impacted on a wedge surface, and then moved from the narrow zone to the wide zone driven by Laplace pressure ${ }^{27,28,36-38}$. Fig. 4 (b) shows moving characteristics at different wedge angle $\alpha$. When $\alpha=4 \sim 12^{\circ}$, all water droplets moved directionally on the wedge surface. Moreover, during the moving process, the droplets were in bulk movement, i.e. spontaneous directional transport of water droplet was realized (Movie S2). However, in most existing reports on realizing droplet transport based on wettability, the droplet spreads in the direction of hydrophilic trajectory, the bulk movement of droplets could not be realized, and some liquids will dissipate along the trajectory ${ }^{25,28}$. With the increase of wedge angle $\alpha$, the maximum speed of droplet increased, and its maximum transport distance increased [Fig. 4 (c)]. When $\alpha=8^{\circ}$, the maximum transport distance of single droplet reached $10.9 \mathrm{~mm}$ with a maximum speed of $16.3 \mathrm{~mm} / \mathrm{s}$, and the transportability was better than that when $\alpha=4^{\circ}$ or $12^{\circ}$.

To analyze the mechanism of the directional transport of water droplet, a theoretical model was developed [Fig. 6 (a)]. When the droplet is moving horizontally, Laplace pressure produces driving force $f_{\text {dri }}$, the friction force $f_{\text {fri }}$ 
between droplet and substrate as well as the drag force $f_{\text {dra }}$ act as the resistance between the droplet and oil layer. Therefore, the total force $F$ can be described as follows:

$$
F=f_{d r i}-f_{f r i}-f_{d r a}
$$

The driving force $f_{\mathrm{dri}}=P \cdot S(x)$, where $S(x)$ is the cross-section area of the droplet, and Laplace pressure $P$ in the $x$ direction of the water droplet on the water harvesting platform with a wedge angle $\alpha$ can be estimated by the Equation (2).

$$
P: \frac{\gamma}{R(x)}: \frac{\gamma}{w(x)} \sin \theta(x)
$$

Where $\gamma$ is the surface tension of water, $R(x)$ denotes the local curvature of the droplet on the water harvesting platform with a wedge angle. $R(x)$ can be described as $R(x)=w(x) / 2 \sin \theta(x)$, and $w(x)$ is the local width of the water harvesting platform, $\theta(x)$ is the local contact angle of the droplet. During the movement process of the droplet, $w(x)$ gradually increases while $\theta(x)$ is slightly changed, which leads to a gradual increase of $R(x)$, and the driving force $f_{\text {dri }}$ will eventually become smaller and smaller. On the other hand, the friction force $f_{\text {fri }}=\mu G$, where $\mu$ is the friction coefficient between droplet and water harvesting platform, $G$ is the gravity of droplet, indicating that the $f_{\text {fri }}$ is approximate a constant. Moreover, the drag force $f_{\text {dra }}=-k R(x) \eta v(x)$, where $k, \eta$, and $v(x)$ are the dimensionless constant, the viscosity of silicone oil and the local velocity of the droplet, respectively. In the early stage of droplet movement, both $R(x)$ and $v(x)$ will gradually increase, which will lead to an increase of the drag force $f_{\text {dra }}$ and results in an increased resistance force to slow down the movement of droplet until it stops. Therefore, the moving distance of a single droplet will be limited, which is consistent with the experimental results [Fig. 4 (c)].

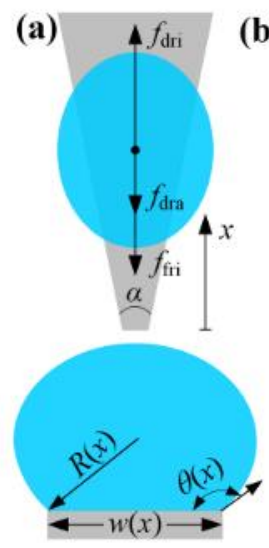

(b)

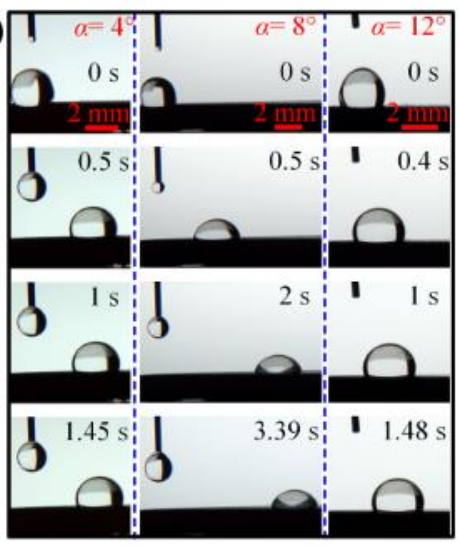

(d)

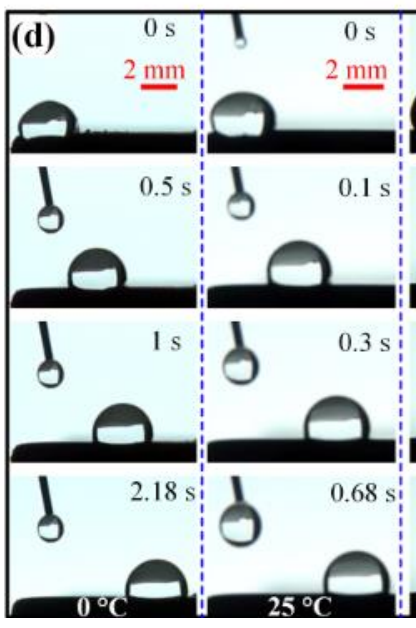

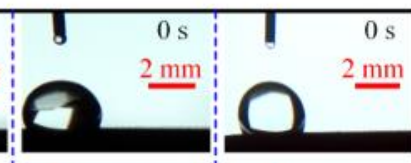
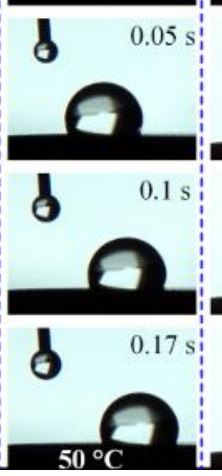
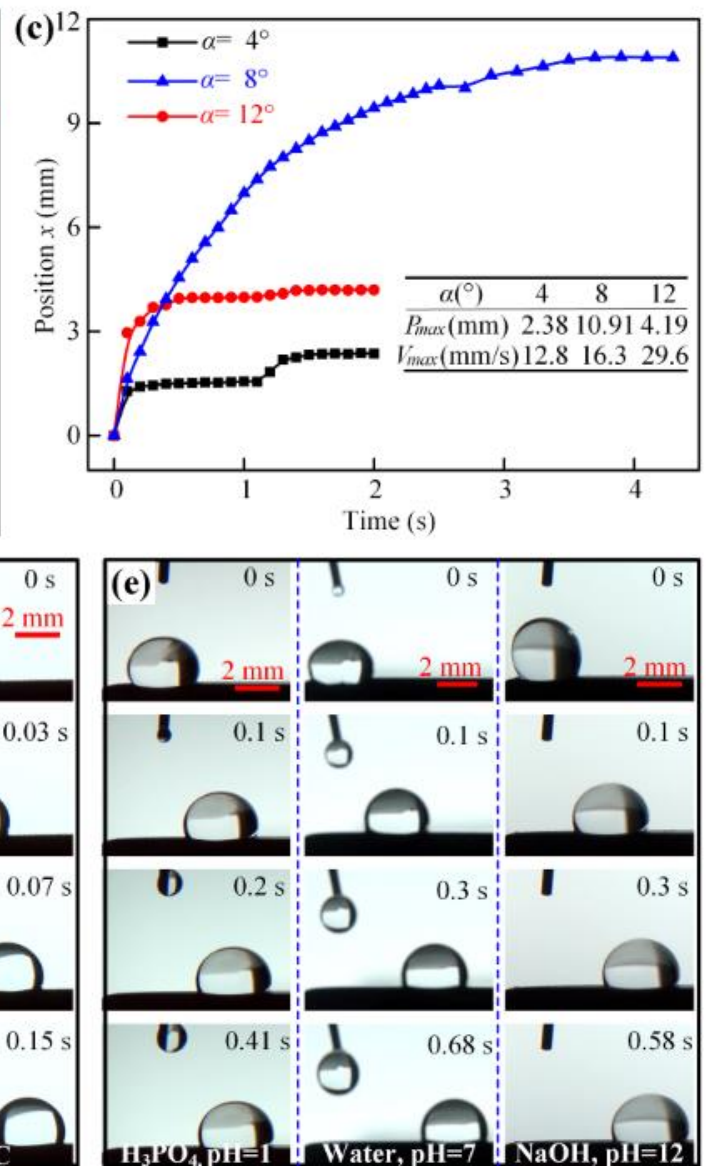

Fig. 4 (a) Schematic of directional transport of water droplet on water harvesting platform. (b) Time-lapse photography (side view) showing the moving process of a droplet on water harvesting platform with different wedge 
angle $\alpha$ of $4^{\circ}, 8^{\circ}$, and $12^{\circ}$. (c) Variation in the moving distances of the droplet with time at different wedge angle $\alpha$. (d) Directional transport of water droplet on the water harvesting platform with a wedge angle of $8^{\circ}$ at a different temperature. (e) Directional transport of droplet with different $\mathrm{pH}$ on the water harvesting platform with a wedge angle of $8^{\circ}$ at $25{ }^{\circ} \mathrm{C}$. The droplet volume was $\sim 5 \mu \mathrm{L}$.

Further study shows that the spontaneously directional transport of water droplets could be realized on water harvesting platforms under dropwise transportation condition at $0{ }^{\circ} \mathrm{C}, 25{ }^{\circ} \mathrm{C}, 50{ }^{\circ} \mathrm{C}$ and $100{ }^{\circ} \mathrm{C}$ [Fig. 4 (c)]. Additionally, the spontaneously directional transport of droplet could be realized for droplets with $\mathrm{pH}$ value ranging from 1 to 12 .

Therefore, the water harvesting platform with a wedge angle served as a transport platform, on which the spontaneously directional transport of droplets was realized under dropwise transportation condition within a temperature range from 0-100 ${ }^{\circ} \mathrm{C}$. During the transport process, water droplets did not wet the water harvesting platform due to its water repellent properties, and this leads to a lossless transport (save for any evaporation). Moreover, the transport of both acid and alkaline drops was realized on the prepared water harvesting platform. However, the transport distance of single droplet was limited, for example, when the wedge angle was $8^{\circ}$, the maximum transport distance was only about $10 \mathrm{~mm}$, which invariably limited the practical application of the water harvesting platform.

To realize long-distance transport of droplet, we proposed to drive the continuous movement of the droplet by converting surface energy to kinetic energy during merging process, as shown in Fig. 5.

When two droplets merge, the surface energy change caused by surface area loss will drive the spontaneous movement of the droplets. ${ }^{39}$ When the droplet radius $R(x)$ was small while the width of the water harvesting platform $w(x)$ was large, the droplet did not move on the water harvesting platform, as shown in Fig. 5 (a). When the droplet was positioned onto the tip of the water harvesting platform, the droplet was driven by Laplace forces to contact and merge with the previously distributed droplet. When two droplets merged, the driving force increased, and the surface energy was then converted into kinetic energy, making the merged droplet moving on to perform the motions of contacting and merging, and this is how the long-term transport of droplets was realized [Fig. 5 (b) and Movie S3]. And the average transport speed in the droplet merging process is about $1.82 \mathrm{~mm} / \mathrm{s}$, while only 0.29 $\mathrm{mm} / \mathrm{s}$ in the droplet chasing process. This is because it takes a lot of time to accumulate droplets to a big one to move on SLIPS during the droplet chasing process [Movie 3]. For single droplet transport, the droplet stopped after being transported over a certain distance and this liquid transport distance was limited. When the droplets were continuously added to the tip of the water harvesting platform, the droplet would be transported for longer-distances in the repeated motions of contacting and merging. Although the length of the transport platform in this experiment is $40 \mathrm{~mm}$, the transport distance is theoretically unrestricted with the increase of droplet volume due to the fact that the diameter of the droplet will continuously grow after contacting and merging with another one. 

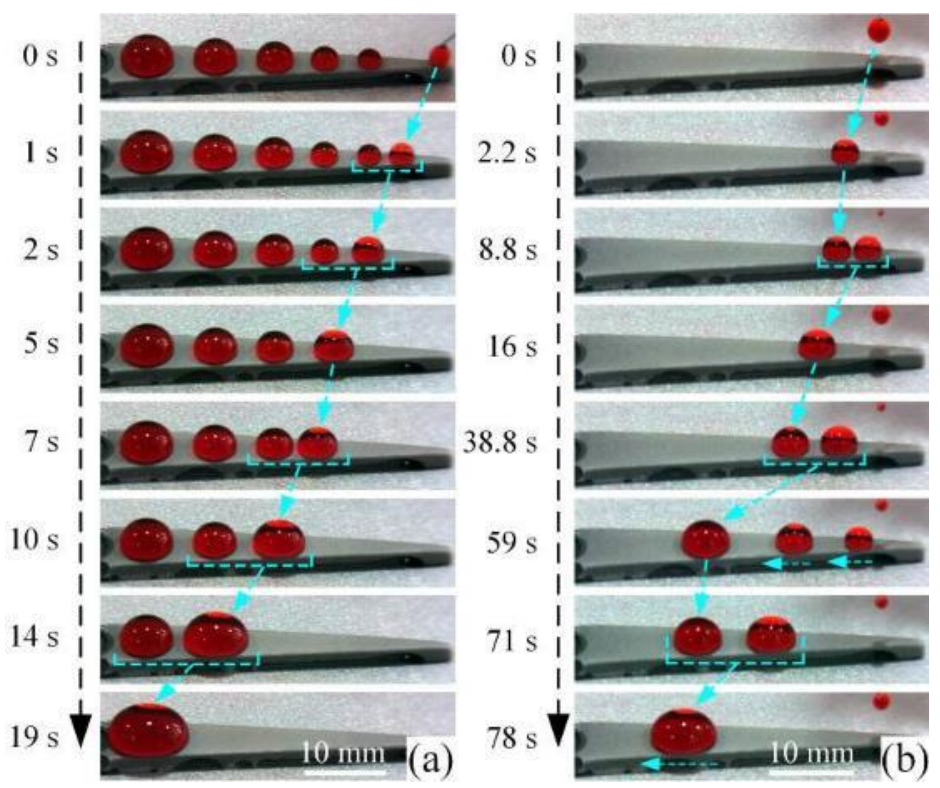

Fig. 5 Directional transport of water droplet (dyed red for visualization) driven by droplet (a) merging and (b) chasing. Length of the transportation region is about $40 \mathrm{~mm}$.

According to the above study, the water harvesting platform can be used to achieve direct and continuous dropwise condensation and water collection in the air at low temperature. The water harvesting platform with a wedge angle can be used to realize spontaneous directional transport of droplets.

\subsection{Both Water Collection and Directional Transport on Water Harvesting Platform}

It should be noted that the water collection property is not the only requirement in some unique situation such as desert beetles, where directional transport of the collected water droplet to the target area is more significant. To transport droplets to target an area after water collection, we designed a water harvesting platform with a length of about $100 \mathrm{~mm}$ consisting of a water collection region, a transportation region and a reservoir region (Fig. 6). The moisture in the air condensed into water droplets on the platform at a temperature of $-2 \sim 1{ }^{\circ} \mathrm{C}$, as shown in Fig. 6 (a). The droplets in the collection region slid down to the transportation region under gravity. Since the droplets in the collection region continuously travelled to the transportation region, the droplets continuously merged and thus sustained directional transport, allowing droplets to finally reach the reservoir region (Movie S4). In the foggy atmosphere, the condensation and transport efficiency of the droplet is significantly higher, as shown in Fig. 6 (b) and Movie S5. Therefore, by taking advantage of dropwise condensation and dropwise transportation characteristics of the substrate, a water harvesting platform was designed to realize water collection and spontaneous directional transport. This novel development water harvesting platform has great potential for application in developing advanced transportation devices, multifunctional sensors, and actuators, etc. 


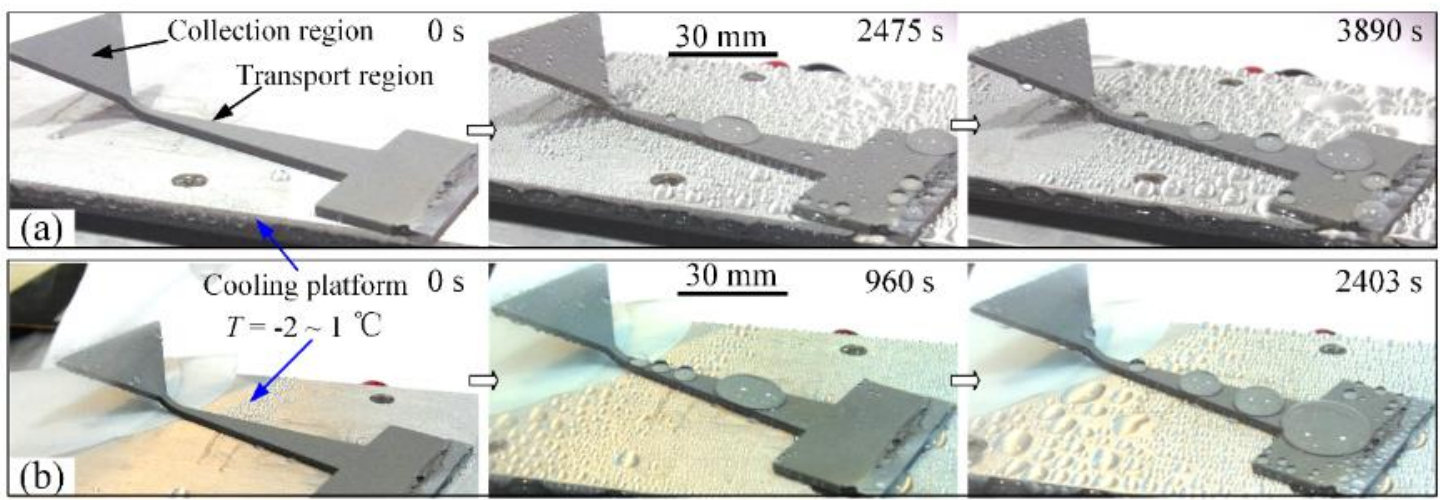

Fig. 6 Continuous condensation and directional transport process on the water harvesting platform in (a) air environment and (b) foggy atmosphere. Length of the water harvesting platform is about $100 \mathrm{~mm}$.

\section{Conclusions}

In summary, we designed a water harvesting platform to achieve stable water condensation from moisture and longdistance directional water transport based on SLIPS at low temperature. Continuous fog harvesting was achieved through dropwise water condensation followed by spontaneous water transport in a repeated motion of water droplet merging and chasing, and this transport was lossless (save evaporation) for the water droplets due to the water repellence of the slippery surfaces. The water harvesting platform is stable and retains its functions upon contacting with acid/alkali droplets and even strong water jet at either low or high temperature. Although the surfaces of the water harvesting platform had ice formation at $-15{ }^{\circ} \mathrm{C}$ after 4 hours, the ice blocks could be easily removed by a small deicing force without leaving any ice residue. This robust water harvesting platform has great potential to be applied for water collection in the air in regions which suffer from water scarcity.

\section{Acknowledgements}

This work was financially supported by National Natural Science Foundation of China (NSFC, Grant No. 51675171 and 51605078), the Fundamental Research Funds for the Central Universities (No. 531107050976), and the Science and Technology Planning Project of Hunan Province (No. 2016TP1008). Y. L. acknowledges the support from EPSRC project EP/N024915/1.

\section{Supplementary data}

The anti-wetting and anti-icing property of SLIPS are available in section S1 and S2.

\section{References:}

[1] J. Ju, X. Yao, S. Yang, L. Wang, R. Sun, Y. He and L. Jiang, Adv. Funct. Mater., 2015, 24, 6933.

[2] M. Cao, J. Ju, K. Li, S. Dou, K. Liu and L. Jiang, Adv. Funct. Mater., 2014, 24, 3235.

[3] J. Ju, H. Bai, Y. Zheng, T. Zhao, R. Fang and L. Jiang, Nat Commun, 2012, 3, 1247.

[4] L. Zhai, M. C. Berg, F. C. Cebeci, Y. Kim, J. M. Milwid, M. F. Rubner and R. E. Cohen, Appl. Phys. Lett., 2015, 6, 1213.

[5] Y. Z, Y. FF, W. Y, Y. L, D. S, L. K, J. L and W. X, Small, 2017, 1701403.

[6] S. J. Hong, C. C. Chang, T. H. Chou, Y. J. Sheng and H. K. Tsao, J.phys.chem.c, 2012, 116, 26487.

[7] A. R. Parker and C. R. Lawrence, Nature, 2001, 414, 33.

[8] Y. Zheng, H. Bai, Z. Huang, X. Tian, F. Q. Nie, Y. Zhao, J. Zhai and L. Jiang, Nature, 2010, 463, 640. 
[9] Y. Tian, P. Zhu, X. Tang, C. Zhou, J. Wang, T. Kong, M. Xu and L. Wang, Nat Commun, 2017, 8, 1080.

[10] H. J. Ensikat, P. Ditschekuru, C. Neinhuis and W. Barthlott, Beilstein J. Nanotech, 2011, $2,152$.

[11] D. Richard, C. Clanet and D. Quéré, Nature, 2002, 417, 811.

[12] T. S. Wong, S. H. Kang, S. K. Y. Tang, E. J. Smythe, B. D. Hatton, A. Grinthal and J. Aizenberg, Nature, 2011, 477, 443.

[13] Y. Lu, S. Sathasivam, J. Song, C. R. Crick, C. J. Carmalt and I. P. Parkin, Science, 2015, 347, 1132.

[14] M. Wang, Q. Liu, H. Zhang, C. Wang, L. Wang, B. Xiang, Y. Fan, C. Guo and S. Ruan, Acs Appl Mater Inter, 2017, 9, 29248.

[15] X. Yang, J. Song, J. Liu, X. Liu and Z. Jin, Sci Rep-Uk, 2017, 7, 8816.

[16] H. Bai, L. Wang, J. Ju, R. Sun, Y. Zheng and L. Jiang, Adv. Mater., 2014, 26, 5025.

[17] K. Yang, K. Lin, C. Tu, Y. He and C. Wang, Int. J. Heat Mass Tran., 2017, 115, 1032.

[18] S. Zhang, J. Huang, Y. Tang, S. Li, M. Ge, Z. Chen, K. Zhang and Y. Lai, Small, 2017, 13, 1600687.

[19] X. Gong, X. Gao and L. Jiang, Adv. Mater., 2017, 1703002.

[20] J. B. Boreyko and C. H. Chen, Phys. Rev. Lett., 2009, 103, 184501.

[21] N. Miljkovic, D. J. Preston, R. Enright and E. N. Wang, Appl. Phys. Lett., 2014, 105, R175.

[22] N. Miljkovic and E. N. Wang, Mrs Bulletin/materials Research Society, 2013, 38, 397.

[23] H. Chen, L. Zhang, Y. Zhang, P. Zhang, D. Zhang and L. Jiang, J. Mater Chem a, 2017, 5, 6914.

[24] C. Li, N. Li, X. Zhang, Z. Dong, H. Chen and L. Jiang, Angew. Chem. Int. Edit., 2016, 55, 14988.

[25] H. Chen, P. Zhang, L. Zhang, H. Liu, Y. Jiang, D. Zhang, Z. Han and L. Jiang, Nature, 2016, 532, 7.

[26] C. Yu, X. Zhu, K. Li, M. Cao and L. Jiang, Adv. Funct. Mater., 2017, 27, 1701605.

[27] C. Zhang, B. Zhang, H. Ma, Z. Li, X. Xiao, Y. Zhang, X. Cui, C. Yu, M. Cao and L. Jiang, Acs Nano, 2018, 12, 2048.

[28] A. Ghosh, R. Ganguly, T. M. Schutzius and C. M. Megaridis, Lab Chip, 2014, 14, 1538.

[29] Y. Hirai, H. Mayama, Y. Matsuo and M. Shimomura, Acs Appl Mater Inter, 2017, 9, 15814.

[30] F. Tseng, K. Lin, H. Hsu and C. Chieng, Sensor. Actuat. A.-Phys., 2004, 111, 107.

[31] M. Cao, K. Li, Z. Dong, C. Yu, S. Yang, C. Song, K. Liu and L. Jiang, Adv. Funct. Mater., 2015, $25,4114$.

[32] H. Geng, H. Bai, Y. Fan, S. Wang and T. Ba, Mater Horiz, 2018,.

[33] J. Li, Y. Hou, Y. Liu, C. Hao, M. Li, M. K. Chaudhury, S. Yao and Z. Wang, Nat Phys, 2016, $12,606$.

[34] Y. Zheng, J. Cheng, C. Zhou, H. Xing, X. Wen, P. Pi and S. Xu, Langmuir, 2017, 17, 4172.

[35] K. Park, P. Kim, A. Grinthal, N. He, D. Fox, J. C. Weaver and J. Aizenberg, Nature, 2016, 531, 78.

[36] S. Huang, J. Song, Y. Lu, F. Chen, H. Zheng, X. Yang, X. Liu, J. Sun, C. J. Carmalt, I. P. Parkin and W. Xu, Acs Appl Mater Inter, 2016, 8, 2942.

[37] J. H. Guan, E. Ruiz-Gutierrez, B. B. Xu, D. Wood, G. McHale, R. Ledesma-Aguilar and G. G. Wells, Soft Matter, 2017, 13, 3404.

[38] Y. Zheng, J. Cheng, C. Zhou, H. Xing, X. Wen, P. Pi and S. Xu, Langmuir, 2017, 33, 4172.

[39] T. Jian, Z. Jie, H. Y. Guo, J. Li, X. Q. Feng and X. Gao, J. Phys Chem Lett, 2014, 5, 2084. 